\title{
Modification of Carbon Paste Electrode with MWCNTs and Benzethonium chloride and its Application as a Novel Sensor for Determination of Benzophenone-4 in Cosmetic Preparations
}

\author{
Hanan M. Elwy ${ }^{1, *}$, Rana S. Sherif ${ }^{l}$, Sawsan M. Amer ${ }^{2}$, G. A. Sedik ${ }^{2, * *}$ \\ ${ }^{1}$ Pharmaceutical Chemistry Department, National Organization of Drug Control and Research.Giza, \\ Egypt. \\ ${ }^{2}$ Analytical Chemistry Department, Faculty of Pharmacy, Cairo University, 11562 \\ Cairo, Egypt. \\ *E-mail: hanan18s@yahoo.com \\ **E-mail: Ghada.sedik@pharma.cu.edu.eg
}

doi: $10.20964 / 2020.05 .21$

Received: 18 December 2019 / Accepted: 25 February 2020 / Published: 10 April 2020

\begin{abstract}
A novel electrochemical sensor is designed for a quick and accurate voltammetric determination of Benzophenone-4 (BZ-4) using a multiwalled carbon nanotube (MWCNTs) electrode in the presence of emulsifying agents. The amounts of surfactant added had a great influence on the voltammetric behavior of the modified carbon paste electrode. The developed voltammetric response of the designed electrode is studied in the existence and absence of Benzethonium chloride (BC) and interesting electrocatalytic effects are found. The increase of the current signal during the oxidation process of BZ-4 is related to the concentration of BZ-4, $\mathrm{pH}$ of the medium, concentration, kind of surfactant and accumulation time at the surface of the electrode. By the aid of scanning electron microscope as well as cyclic voltammetry, the electrochemical inspection in addition to the characterization of the modified electrode were conducted. Benzethonium chloride (BC) is used in the quantification of BZ-4 in cyclic and square-wave voltammetry at multiwalled carbon nanotubes (MWCNTs) modified electrode. It was found to enhance the peak current from $46.4 \mu \mathrm{A}$ in deprivation of surfactant to $60.4 \mu \mathrm{A}$ when present. Peak current response obtained for BZ-4 under optimum experimental conditions is linear within a range of $6.0 \times 10^{-9}$ to $1.09 \times 10^{-4} \mathrm{~mol} \mathrm{~L}^{-1}$ with correlation coefficients of 0.9995 . The limit of detection is $3.3 \times 10^{-9} \mathrm{~mol} \mathrm{~L} \mathrm{~L}^{-1}$. The modified electrode displays high sensitivity and reproducibility which enables its utilization for quantification of BZ-4 in its pharmaceutical formulations.
\end{abstract}

Keywords: Square wave voltammetry, Benzethonium chloride, Benzophenone-4.

\section{FULL TEXT}


(C) 2020 The Authors. Published by ESG (www.electrochemsci.org). This article is an open access article distributed under the terms and conditions of the Creative Commons Attribution license (http://creativecommons.org/licenses/by/4.0/). 